'Centro de Salud Familiar de Los Lagos, Región de Los Ríos, Chile. 2Departamento Ciencias de la Actividad Física, Universidad de Los Lagos, Osorno, Chile.

${ }^{3}$ Laboratorio de Ciencias del Ejercicio, Clínica MEDS, Las Condes, Chile. ${ }^{4}$ Instituto DUOC, Pontificia Universidad Católica, Santiago, Chile. ${ }^{5}$ Facultad de Educación, Ciencias Sociales y Humanidades, Departamento de Educación Física, Universidad de la Frontera, Temuco, Chile. aProfesor de Educación Física, Lic., MSc. Entrenamiento Deportivo. bProfesor de Educación Física, Lic., PhD. Fisiología del Ejercicio. 'Tecnólogo en Deportes, Lic., MSc Cs. Biológicas.

dProfesor de Educación Física, Lic., MSc. Medicina y Cs del Deporte.

Profesora de Educación Física, MSc. PhD Motricidad Humana. Profesor de Educación Física, MSc. Motricidad Humana.

Financiamiento: Este proyecto de investigación fue financiado con Fondos del Departamento de Salud Pública Municipal de la llustre Municipalidad de Los Lagos y por aportes privados. No se declaran conflicto de intereses entre las fuentes de financiamiento en el diseño del presente estudio

Recibido el 24 de diciembre de 2013 aceptado el 15 de abril de 2014

Correspondencia a: MSc., Cristian Álvarez Lepín Prat \#162

Centro de Salud Familiar Los Lagos Comuna de Los Lagos Región de Los Ríos, Chile. Fono: 063-2-460304 profecristian.alvarez@gmail.com

\section{¿Pueden ocho semanas de ejercicio físico combinado normalizar marcadores metabólicos de sujetos hiperglicémicos y dislipidémicos?}

\author{
CRISTIAN ÁLVAREZ ${ }^{1, a}$, RODRIGO RAMÍREZ-CAMPILLO ${ }^{2, b}$, \\ CARLOS HENRÍQUEZ-OLGUIIN ${ }^{3, \mathrm{c}}$, MAURICIO CASTRO-SEPÚLVEDA ${ }^{4, \mathrm{~d}}$, \\ VANESA CARRASCO ${ }^{5, \mathrm{e}}$, CRISTIAN MARTÍNEZ $^{6, \mathrm{f}}$
}

\section{Eight weeks of combined high intensity intermittent exercise normalized altered metabolic parameters in women}

Background: Short term physical training programs may improve insulin resistance and hyperglycemia. Aim: To assess the effects of eight weeks of combined exercise program on serum lipids and glycemic level in women with hyperglycemia and hypercholesterolemia. Patients and Methods: Ten healthy women, nine women with hyperglycemia, ten with hypercholesterolemia and nine with hyperglycemia/hypercholesterolemia were studied. Participants were subjected to eight weeks into a program of combined physical exercise (high intensity interval + resistance training). Results: Fasting glycemia decreased by 12 and 14\% in hyperglycemic and hyperglycemic/hypercholesterolemic participants, respectively. Serum insulin decreased in all groups in a range from 27 to $37 \%$. HOMA $I R$ for insulin resistance decreased similarly. A significant decrease in TC and TG was observed only in those altered baseline subjects. Conclusions: Eight weeks of combined physical exercise had a favorable effect on insulin resistance in this group of women.

(Rev Med Chile 2014; 142: 458-466)

Key words: Exercise; Insulin resistance; Physical education and training.
L a insulino resistencia (IR) se caracteriza por una disminución relativa en la habilidad de la insulina para ejercer sus efectos sobre la glucosa y el metabolismo de lípidos en el hígado, tejido adiposo y músculo ${ }^{1}$. La IR conlleva a diabetes mellitus tipo $2(\mathrm{DT} 2)^{2}$ relacionándose esta última enfermedad, con una serie de otras alteraciones de alta prevalencia como la hipercolesterolemia y dislipidemias ${ }^{3}$, usualmente asociados a sobrepeso/obesidad ${ }^{4}$, y en donde el ejercicio físico (EF) ha demostrado jugar un importante rol en su prevención y terapia ${ }^{5,6}$. Si bien la IR y DT2 podrían relacionarse con factores genéticos ${ }^{1,2}$ y el envejecimiento ${ }^{7}$, la inactividad física tendría una relación directa con estas enfermedades ${ }^{8}$.

Actualmente existen potentes reportes acerca de los beneficios de algunas metodologías de EF con la característica de alta intensidad y muy corta duración que incrementan la insulino sensibilidad $^{9}$, mejoran el control glicémico ${ }^{10} \mathrm{y}$ que producen una mayor oxidación de lípidos durante y después de ejercicio ${ }^{11}$, como el ejercicio intermitente de alta intensidad (HIT) "del inglés high intensity interval training",10,12-14. Por otra parte, también se han dado a conocer un número importante de programas de ejercicio físico de 
sobrecarga (EFS) que mejoran la funcionalidad muscular ${ }^{15}$, el control glicémico ${ }^{16}$ y la composición corporal ${ }^{17}$ en estos pacientes.

Previamente hemos reportado los efectos de 12 semanas de HIT y/o EFS, donde observamos que ambas metodologías mejoraron el control glicémico de pacientes pre-diabéticos ${ }^{14} \mathrm{y}$ donde también la metodología HIT mejora el perfil de lípidos de sujetos levemente hipercolesterolémi$\cos ^{18}$. En Chile no existen estudios de intervención aplicando ejercicio de tipo HIT de tiempo menor a 12 semanas en marcadores metabólicos como el $\mathrm{HOMA}_{\mathrm{IR}}$ y lipoproteínas plasmáticas frecuentemente utilizadas en salud pública, y existe poca información del ejercicio HIT aplicado en conjunto con ejercicio de fuerza en pacientes con alteraciones metabólicas. El objetivo del presente estudio fue investigar los efectos de un programa corto de 8 semanas de ejercicio físico combinado (HIT + EFS) en los niveles de IR y lípidos plasmáticos de mujeres sanas, con hiperglicemia e hipercolesterolemia. Un objetivo adicional fue analizar los efectos de la intervención en la composición corporal, presión arterial y rendimiento físico aeróbico.

\section{Métodos}

En un estudio cuasi-experimental, 56 mujeres con bajo nivel de actividad física (menos de 150 min de actividad física por semana ${ }^{19}$ ), pertenecientes al centro de salud familiar (CESFAM) de Los Lagos, Región de Los Ríos, Chile, fueron voluntarias para participar de un programa de EF combinado. Sólo treinta y ocho $(\mathrm{n}=38)$ sujetos fueron incluídos en los análisis estadísticos finales, siendo 18 excluidos por diversos motivos ( 5 por enfermedad viral repentina, 6 por no cumplir con todas las evaluaciones iniciales, y 7 por tener $\leq 80 \%$ de adherencia a las sesiones de intervención). De acuerdo a criterios de clasificación estándar ${ }^{3}$, los sujetos fueron asignados a uno de cuatro grupos; hiper-glicémicos ( $\mathrm{HG}, \mathrm{n}$ $=9$ ), hiper-colesterolémicos ( $\mathrm{HC}, \mathrm{n}=10)$, hiperglicémicos/hiper-colesterolémicos (HG/HC, $\mathrm{n}=$ 9) y sujetos sanos (SANOS, $n=10$ ), que también sirvieron de grupo control. Las características basales de los sujetos se presentan en la (Tabla 1).

Los criterios de inclusión fueron: Ser inactiva físicamente ( $\leq 150 \mathrm{~min}$ de EF/semana), índice de masa corporal (IMC) entre 25 a $35 \mathrm{~kg} / \mathrm{m}^{2}$, glicemia $<100 \mathrm{mg} / \mathrm{dl}$ (normo-glicémicos) y 100 a $126 \mathrm{mg} /$ $\mathrm{dl}$ (hiper-glicémicos) ${ }^{20}$, colesterol total $(\mathrm{CT})<200$ $\mathrm{mg} / \mathrm{dl}$ (normo-colesterolémicos) y $>200 \mathrm{mg} / \mathrm{dl}$ (hiper-colesterolémicos) ${ }^{21}$, con control médico y electrocardiograma actualizado.

Los criterios de exclusión fueron: Presencia de enfermedad isquémica, arritmia, taquicardia, asma y/o EPOC, utilización drogas o medicación con efecto sobre la masa corporal o el control metabólico. El estudio fue desarrollado considerando la Declaración de Helsinki y fue aprobado por el comité de ética del CESFAM de Los Lagos, Chile.

\section{Evaluación de la composición corporal}

La masa corporal se midió con una balanza digital con precisión de $0,1 \mathrm{~kg}\left(\mathrm{OMRON}^{\oplus}\right.$, Model HBF-INT). La talla se midió con un estadiómetro profesional (marca Health o Meter ${ }^{\oplus}$, USA), con precisión de $0,1 \mathrm{~cm}$. El IMC se calculó en base a la masa corporal dividido por la talla al cuadrado $\left(\mathrm{kg} / \mathrm{m}^{2}\right)$. El perímetro de cintura se midió con una cinta métrica con una precisión de $0,1 \mathrm{~cm}$ (marca Hoechstmass ${ }^{\circledR}$ West Germany). Adicionalmente se midieron 4 pliegues de tejido adiposo subcutáneo (pliegue tricipital, subescapular, cresta iliaca y pierna), de acuerdo a protocolos descritos previamente $^{22}$.

\section{Evaluación de la presión arterial y rendimiento físico aeróbico}

La presión arterial sistólica (PAS) y diastólica (PAD) se midió con un monitor digital (marca $\mathrm{OMRON}^{\circledR}$, modelo HEM 7114) entre las 8 y $11 \mathrm{~h}$ am, durante tres oportunidades en días no consecutivos, mientras los sujetos permanecían sentados 15 min previos a cada medición. Se aplicó el test de Urho Kaleka Kekkonen (TUKK), consistente en caminar en el menor tiempo posible una distancia de $2 \mathrm{~km}$ en superficie plana. El test se realizó en un recinto cerrado de amplias dimensiones, utilizando un reloj cardiómetro (Polar ${ }^{\circledR}$, modelo RS400, Finlandia) para registrar la frecuencia cardiaca en reposo (FCR), y la misma durante y después del test, el cual se llevó a cabo considerando el protocolo original ${ }^{23} \mathrm{y}$ al validado en la población Chilena $^{24}$ (Tabla 2).

\section{Programa de ejercicio físico combinado}

Posterior a dos semanas de adaptación al ejercicio (6 sesiones), los cuatro grupos de sujetos 
Tabla 1. Cambios en la composición corporal después de 8 semanas de un programa de ejercicio físico combinado en mujeres sanas y con diferentes alteraciones metabólicas

\begin{tabular}{|c|c|c|c|c|c|c|}
\hline Variable & Test & $\begin{array}{c}\text { HG }^{\mathrm{a}} \\
(n=9)\end{array}$ & $\begin{array}{c}H C^{b} \\
(n=10)\end{array}$ & $\begin{array}{l}\text { HG/HCc } \\
(n=9)\end{array}$ & $\begin{array}{l}\text { SANOS }^{d} \\
(n=10)\end{array}$ & $\mathbf{P}_{\text {interaction }}$ \\
\hline Edad & Pre $_{0}$ & $38 \pm 9,8$ & $42 \pm 8,4$ & $42 \pm 8,2$ & $48 \pm 8,8$ & 0,716 \\
\hline Talla (m) & Pre $_{0}$ & $1,55 \pm 0,03$ & $1,59 \pm 0,06$ & $1,53 \pm 0,06$ & $1,54 \pm 0,03$ & 0,093 \\
\hline Peso $(\mathrm{kg})$ & $\begin{array}{l}\text { Pre }_{0} \\
\text { Post }_{8} \\
\triangle \%\end{array}$ & $\begin{array}{c}73,7 \pm 13,3 \\
72,6 \pm 12,8 \\
-1,5\end{array}$ & $\begin{array}{c}75,7 \pm 8,1 \\
73,9 \pm 7,7^{* *} \\
-2,4(p=0,010)\end{array}$ & $\begin{array}{c}73,9 \pm 9,1 \\
71,6 \pm 8,8^{*} \\
-3,1(p=0,012)\end{array}$ & $\begin{array}{c}70,4 \pm 8,1 \\
68,1 \pm 7,9^{*} \\
-3,2(p=0,013)\end{array}$ & 0,674 \\
\hline IMC $\left(\mathrm{kg} / \mathrm{m}^{2}\right)$ & $\begin{array}{l}\text { Pre }_{0} \\
\text { Post }_{8} \\
\triangle \%\end{array}$ & $\begin{array}{c}30,9 \pm 5,3 \\
30,4 \pm 5,1 \\
-1,5\end{array}$ & $\begin{array}{c}30,2 \pm 3,7 \\
29,5 \pm 3,6^{* *} \\
-2,4(p=0,010)\end{array}$ & $\begin{array}{c}31,6 \pm 2,5 \\
30,6 \pm 2,2^{* *} \\
-3,1(p=0,010)\end{array}$ & $\begin{array}{c}29,8 \pm 4,0 \\
28,8 \pm 3,8^{* *} \\
-3,2(p=0,010)\end{array}$ & 0,767 \\
\hline Perímetro de cintura $(\mathrm{cm})$ & $\begin{array}{l}\text { Pre }_{0} \\
\text { Post }_{8} \\
\triangle \%\end{array}$ & $\begin{array}{c}104,6 \pm 13,0 \\
100,5 \pm 12,7^{* *} \\
-3,9(p=0,01)\end{array}$ & $\begin{array}{c}101,1 \pm 8,0 \\
98,3 \pm 10,0 \\
-2,9\end{array}$ & $\begin{array}{c}100,7 \pm 9,7 \\
98,2 \pm 6,3 \\
-2,0\end{array}$ & $\begin{array}{c}99,5 \pm 8,2 \\
96,3 \pm 7,7 \\
-3,2\end{array}$ & 0,715 \\
\hline Pliegue tricipital (mm) & $\begin{array}{l}\text { Pre }_{0} \\
\text { Post }_{8} \\
\triangle \%\end{array}$ & $\begin{array}{c}34,3 \pm 7,1 \\
33,7 \pm 6,7 \\
-1,7\end{array}$ & $\begin{array}{c}34,3 \pm 6,8 \\
32,9 \pm 6,3 \\
-3,9\end{array}$ & $\begin{array}{c}37,9 \pm 10,1 \\
36,0 \pm 8,3 \\
-2,8\end{array}$ & $\begin{array}{c}30,8 \pm 6,1 \\
31,7 \pm 8,5 \\
+2,3\end{array}$ & 0,270 \\
\hline Pliegue subescapular (mm) & $\begin{array}{l}\text { Pre }_{0} \\
\text { Post }_{8} \\
\triangle \%\end{array}$ & $\begin{array}{c}41,9 \pm 10,6 \\
36,7 \pm 11,8 \\
-14\end{array}$ & $\begin{array}{c}41,2 \pm 10,8 \\
36,6 \pm 10,4 \\
-11,5\end{array}$ & $\begin{array}{c}47,0 \pm 11,5 \\
42,5 \pm 7,0 \\
-3,5\end{array}$ & $\begin{array}{c}40,7 \pm 7,4 \\
35,6 \pm 5,5 \\
-11,4\end{array}$ & 0,517 \\
\hline Pliegue cresta iliaca (mm) & $\begin{array}{l}\text { Pre }_{0} \\
\text { Post }_{8} \\
\triangle \%\end{array}$ & $\begin{array}{c}43,8 \pm 12,5 \\
39,7 \pm 11,2 \\
-9,1\end{array}$ & $\begin{array}{c}40,3 \pm 7,9 \\
35,1 \pm 7,1^{*} \\
-12,3(p=0,05)\end{array}$ & $\begin{array}{c}41,6 \pm 7,6 \\
37,8 \pm 6,2 \\
-7,7\end{array}$ & $\begin{array}{c}36,6 \pm 3,3 \\
35,9 \pm 6,4 \\
-2\end{array}$ & 0,310 \\
\hline Pliegue pierna (mm) & $\begin{array}{l}\text { Pre }_{0} \\
\text { Post }_{8} \\
\triangle \%\end{array}$ & $\begin{array}{c}28,3 \pm 6,6 \\
26,3 \pm 4,5 \\
-4,9\end{array}$ & $\begin{array}{c}27,0 \pm 7,8 \\
26,3 \pm 7,5 \\
-2,2\end{array}$ & $\begin{array}{c}27,7 \pm 7,6 \\
28,1 \pm 6,0 \\
-5,7\end{array}$ & $\begin{array}{c}26,5 \pm 5,7 \\
26,9 \pm 6,3 \\
\quad+1,1\end{array}$ & 0,948 \\
\hline
\end{tabular}

Los datos son presentados como media \pm desviación estándar. Grupos son descritos como; (HG): hiperglicémicos, (HC): hipercolesterolémicos, (HG/HC): hiperglicémicos/hipercolesterolémicos y (SANOS): sujetos sanos o también control. Variables son descritas como; (IMC): índice de masa corporal, $\left(\right.$ Pre $\left._{0}\right)$ : antes de intervención y $\left(\right.$ Post $\left._{8}\right)$ : después de intervención. ${ }^{*}$ indica

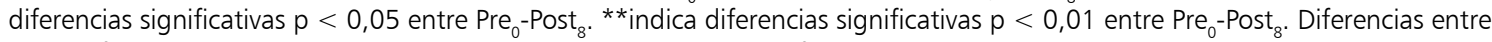
grupos fueron analizadas con ANOVA de 1 vía, mientras que las diferencias en el tiempo en cada grupo mediante ANOVA de medidas repetidas (grupos $x$ tiempo).

completaron 8 semanas de intervención con EF combinado, consistente en realizar en cada sesión ejercicio tipo HIT (17-29 min) + EFS (24 min), completando $\leq 60 \mathrm{~min} /$ sesión. Durante el HIT se completan 8-14 intervalos por sesión, con una intensidad equivalente al $80-100 \%$ de la frecuencia cardiaca de reserva (FCR), de acuerdo al método propuesto por Karvonen ${ }^{25} \mathrm{y}$ controlada mediante pulsómetro marca (Polar ${ }^{\circledR}$, modelo RS400, Finlandia). Entre cada serie de trabajo se completaba $1,15-2$ min de pausa activa, pedaleando a $<70 \%$ FCR. Durante el EFS se utilizaron pesos libres, y se entrenaron 4 grupos musculares (flexores de codo, extensores de codo, flexores de hombro y subescapulares). Se realizaban 3 series para cada grupo muscular, con 1 min de duración cada una, hasta alcanzar la "fatiga muscular". Entre cada serie los sujetos caminaban erguidos durante $1 \mathrm{~min}$. Todas las sesiones de intervención se realizaron entre 8 y $11 \mathrm{~h}$ am. El programa se llevó a cabo en centro de acondicionamiento físico y salud del CESFAM Los Lagos. La adherencia al programa de acuerdo a los grupos fue buena (HG 89, HC 93, HG/HC 86, SANOS 85\%). Las características del programa de EF combinado se presentan en (Tabla 3). 
Ejercicio físico combinado mejora el control metabólico - C. Álvarez et al

Tabla 2. Cambios en variables cardiovasculares y en el rendimiento físico aeróbico, después de 8 semanas de ejercicio físico combinado en mujeres sanas y con diferentes alteraciones metabólicas

\begin{tabular}{|c|c|c|c|c|c|c|}
\hline Variable & Test & $\begin{array}{c}H^{\prime} \mathbf{a}^{\mathbf{a}} \\
(n=9)\end{array}$ & $\begin{array}{c}H C^{b} \\
(n=10)\end{array}$ & $\begin{array}{l}\text { HG/HCc } \\
(n=9)\end{array}$ & $\begin{array}{l}\text { SANOS }^{d} \\
(n=10)\end{array}$ & $\mathbf{P}_{\text {interaction }}$ \\
\hline PAS (mm/Hg) & $\begin{array}{l}\mathrm{Pre}_{0} \\
\text { Post }_{8} \\
\triangle \%\end{array}$ & $\begin{array}{c}123 \pm 6 \\
120 \pm 5 \\
-3,0\end{array}$ & $\begin{array}{c}122 \pm 8 \\
111 \pm 20^{*} \\
-9,0(p=0,004)\end{array}$ & $\begin{array}{c}125 \pm 6 \\
122 \pm 4 \\
-2,0\end{array}$ & $\begin{array}{c}125 \pm 5 \\
122 \pm 5 \\
-2,0\end{array}$ & 0,706 \\
\hline PAD $(\mathrm{mm} / \mathrm{Hg})$ & $\begin{array}{l}\text { Pre }_{0} \\
\text { Post }_{8} \\
\triangle \%\end{array}$ & $\begin{array}{c}70 \pm 8 \\
69 \pm 7 \\
-1,3\end{array}$ & $\begin{array}{c}76 \pm 16 \\
70 \pm 6 \\
-5,3\end{array}$ & $\begin{array}{c}71 \pm 10 \\
69 \pm 9 \\
-1,9\end{array}$ & $\begin{array}{c}69 \pm 9 \\
67 \pm 8^{* *} \\
-2,8(p=0,009)\end{array}$ & 0,545 \\
\hline FCR (lat/min) & $\begin{array}{l}\mathrm{Pre}_{0} \\
\mathrm{Post}_{8} \\
\triangle \%\end{array}$ & $\begin{array}{c}71 \pm 11 \\
71 \pm 9 \\
0\end{array}$ & $\begin{array}{c}79 \pm 3 \\
77 \pm 3^{* *} \\
-2,1(p=0,010)\end{array}$ & $\begin{array}{c}73 \pm 8 \\
72 \pm 7 \\
-0,8\end{array}$ & $\begin{array}{c}74 \pm 5 \\
74 \pm 4 \\
-1,0\end{array}$ & 0,147 \\
\hline TUKK (min) & $\begin{array}{l}\text { Pre }_{0} \\
\text { Post }_{8} \\
\triangle \%\end{array}$ & $\begin{array}{c}21,52 \pm 1,1 \\
19,01 \pm 0,9^{* *} \\
-12,4(p=0,007)\end{array}$ & $\begin{array}{c}22,0 \pm 1,3 \\
19,2 \pm 1,4^{* *} \\
-12,6(p=0,007)\end{array}$ & $\begin{array}{c}22,49 \pm 0,9 \\
19,37 \pm 0,8^{* *} \\
-13,9(p=0,005)\end{array}$ & $\begin{array}{c}22,13 \pm 0,8 \\
18,57 \pm 0,6^{* *} \\
-16(p=0,005)\end{array}$ & 0,493 \\
\hline
\end{tabular}

Los datos son presentados como media \pm desviación estándar. Grupos son descritos como; (HG): hiperglicémicos, (HC): hipercolesterolémicos, (HG/HC): hiperglicémicos/hipercolesterolémicos y (SANOS): sujetos sanos o también control. Variables son descritas como; (TUKK): test $2 \mathrm{~km}$ de caminata, $\left(\right.$ Pre $\left._{0}\right)$ : antes de intervención y (Post ${ }_{8}$ ): después de intervención. *indica diferencias significativas $p<0,05$ entre Pre $_{0}$-Post ${ }_{8}{ }^{* *}$ indica diferencias significativas $p<0,01{\text { entre } \text { Pre }_{0} \text {-Post }}_{8}$.

Tabla 3. Descripción de un programa de 8 semanas de intervención utilizando ejercicio físico combinado en sujetos con sanos y con alteraciones metabólicas

\begin{tabular}{|c|c|c|c|c|c|c|c|c|}
\hline \multirow[t]{2}{*}{ Variable } & \multicolumn{2}{|c|}{ Semana 1-2 } & \multicolumn{2}{|c|}{ Semana 3-4 } & \multicolumn{2}{|c|}{ Semana 5-6 } & \multicolumn{2}{|c|}{ Semana 7-8 } \\
\hline & HIT & EFS & HIT & EFS & HIT & EFS & HIT & EFS \\
\hline Intensidad & $\begin{array}{c}80-100 \% \\
\text { FCR }\end{array}$ & $\begin{array}{l}\text { "Fatiga } \\
\text { muscular" }\end{array}$ & $\begin{array}{c}80-100 \% \\
\text { FCR }\end{array}$ & $\begin{array}{c}\text { "Fatiga } \\
\text { muscular" }\end{array}$ & $\begin{array}{c}80-100 \% \\
\text { FCR }\end{array}$ & $\begin{array}{l}\text { "Fatiga } \\
\text { muscular" }\end{array}$ & $\begin{array}{c}80-100 \% \\
\text { FCR }\end{array}$ & $\begin{array}{c}\text { "Fatiga } \\
\text { muscular" }\end{array}$ \\
\hline $\begin{array}{l}\text { Tiempo de } \\
\text { intervalo de } \\
\text { trabajo (seg) }\end{array}$ & 60 & 60 & 60 & 60 & 60 & 60 & 60 & 60 \\
\hline $\begin{array}{l}\text { Tiempo de } \\
\text { recuperación } \\
\text { (min: seg) }\end{array}$ & 2 & 1 & 1,45 & 1 & 1,30 & 1 & 1,15 & 1 \\
\hline $\begin{array}{l}\text { Método de } \\
\text { recuperación }\end{array}$ & $\begin{array}{c}\text { Pedalear a } \\
\text { carga baja } \\
\text { a } \leq 70 \% \\
\text { FCR }\end{array}$ & $\begin{array}{l}\text { Descanso } \\
1 \text { min en } \\
\text { posición } \\
\text { "erguido" }\end{array}$ & $\begin{array}{c}\text { Pedalear a } \\
\text { carga baja } \\
a \leq 70 \% \\
\text { FCR }\end{array}$ & $\begin{array}{l}\text { Descanso } \\
1 \text { min en } \\
\text { posición } \\
\text { "erguido" }\end{array}$ & $\begin{array}{c}\text { Pedalear a } \\
\text { carga baja } \\
a \leq 70 \% \\
\text { FCR }\end{array}$ & $\begin{array}{l}\text { Descanso } \\
1 \text { min en } \\
\text { posición } \\
\text { "erguido" }\end{array}$ & $\begin{array}{c}\text { Pedalear a } \\
\text { carga baja } \\
\text { a } \leq 70 \% \\
\text { FCR }\end{array}$ & $\begin{array}{l}\text { Descanso } \\
1 \text { min en } \\
\text { posición } \\
\text { "erguido" }\end{array}$ \\
\hline $\begin{array}{l}\text { n total intervalos } \\
\text { de trabajo }\end{array}$ & 8 & 12 & 10 & 12 & 12 & 12 & 14 & 12 \\
\hline $\begin{array}{l}\text { Volumen/día } \\
\text { (min) }\end{array}$ & 17 & 24 & 21 & 24 & 25 & 24 & 29 & 24 \\
\hline $\begin{array}{l}\text { Volumen/sem } \\
\text { (min) }\end{array}$ & 4 & & & & & & & \\
\hline
\end{tabular}

Metodologías de ejercicio son descritas como; (HIT): ejercicio intermitente de alta intensidad, (EFS): ejercicio de fuerza con sobrecarga. Unidades de medida son descritas como; (\%FCR): porcentaje de frecuencia cardiaca de reserva, calculada mediante la fórmula = frecuencia cardiaca de reposo (lat/min) - frecuencia cardiaca máxima (lat/min) de acuerdo a Karvonen y cols, ${ }^{25}$, (min): minutos, (seg): segundos. 


\section{Evaluación de glicemia, insulina y lípidos}

Posterior a $12 \mathrm{~h}$ de ayuno, se obtuvieron muestras $(4 \mathrm{ml})$ de sangre entre las 8 y $10 \mathrm{am}$, desde donde se midieron los niveles de glicemia e insulina, las cuales se depositaron de inmediato en un tuvo con solución anticoagulante. Utilizando la glucosa e insulina en ayunas se obtuvo el valor de $\mathrm{HOMA}_{\mathrm{IR}}$, de acuerdo a la fórmula propuesta por Matthews y cols ${ }^{26} \mathrm{HOMA}_{\mathrm{IR}}=$ glicemia en ayuna $(\mathrm{mg} / \mathrm{dl}) \mathrm{x}$ insulina en ayuna (mUI/ $\mathrm{ml} / 405$. Las muestras fueron centrifugadas a 3.000 r.p.m. durante $15 \mathrm{~min}$ a $-20^{\circ} \mathrm{C}$. La glucosa, el colesterol total (y sus fracciones) y los triglicéridos fueron analizados por métodos estándar, similar a otros estudios ${ }^{14}$. Se analizó la glicemia a través del método enzimático con la técnica glucosa-oxidasa (Trinder, Genzyme Diagnostics, Canada). El colesterol total (CT), triglicéridos y el colesterol HDL (C-HDL) se analizaron mediante el método enzimático calorimétrico
(Diagnostica mbh, Alemania). C-LDL colesterol se calculo con la fórmula de Friedewald ${ }^{27}$. Al finalizar la intervención, las mediciones plasmáticas se realizaron $72 \mathrm{~h}$ después de la última sesión de EF combinado.

\section{Análisis estadístico}

Se utilizó media \pm desviación estándard para describir las variables cuantitativas. Se utilizó el test de Shapiro-Wilk para determinar la normalidad de los datos y el test de Levene para establecer la homocedasticidad. Se aplicó análisis ANOVA de medidas repetidas (grupos $\mathrm{x}$ tiempo), así como el post hoc de Bonferroni para localizar las diferencias entre los tiempo de medición $\left(\mathrm{Pre}_{0}-\mathrm{Post}_{8}\right.$ semanas) y/o entre los grupos. Para las variables no paramétricas se aplicó el test ANOVA de KruskalWallis y el post hoc de Dunn's para comparaciones múltiples. Se aplicó el coeficiente de correlación $r$ de Pearson y Spearman para determinar la re-

Tabla 4. Modificación de variables plasmáticas después de 8 semanas de ejercicio físico combinado en sujetos sanos y con diferentes alteraciones metabólicas

\begin{tabular}{|c|c|c|c|c|c|c|}
\hline Variable & Test & $\begin{array}{c}H^{H G^{a}} \\
(n=9)\end{array}$ & $\begin{array}{c}H C^{b} \\
(n=10)\end{array}$ & $\begin{array}{l}\text { HG/HCc } \\
(n=9)\end{array}$ & $\begin{array}{l}\text { SANOS }^{d} \\
(n=10)\end{array}$ & $\mathbf{P}_{\text {interaction }}$ \\
\hline Glicemia (mg/dl) & $\begin{array}{l}\text { Pre }_{0} \\
\text { Post }_{8} \\
\triangle \%\end{array}$ & $\begin{array}{c}106 \pm 6 \\
93 \pm 4^{* *} \\
-12(p=0,01)\end{array}$ & $\begin{array}{c}94 \pm 8 \\
89 \pm 5 \\
-6\end{array}$ & $\begin{array}{c}107 \pm 5 \\
92 \pm 6^{* *} \\
-14(p=0,01)\end{array}$ & $\begin{array}{c}93 \pm 6 \\
89 \pm 5 \\
-5\end{array}$ & 0,0001 \\
\hline Insulina ( $\mu \mathrm{UI} / \mathrm{ml})$ & $\begin{array}{l}\text { Pre }_{0} \\
\text { Post }_{8} \\
\triangle \%\end{array}$ & $\begin{array}{c}13,6 \pm 4,7 \\
9,1 \pm 3,2^{*} \\
-30,1(p=0,05)\end{array}$ & $\begin{array}{c}13,9 \pm 7,3 \\
8,5 \pm 4,2^{* *} \\
-37(p=0,01)\end{array}$ & $\begin{array}{c}17,2 \pm 7,5 \\
12,7 \pm 6,0^{* *} \\
-26,7(p=0,01)\end{array}$ & $\begin{array}{c}10,1 \pm 3,1 \\
7,4 \pm 3,3^{*} \\
-26,5(p=0,05)\end{array}$ & 0,0970 \\
\hline $\mathrm{CT}(\mathrm{mg} / \mathrm{dl})$ & $\begin{array}{l}\text { Pre }_{0} \\
\text { Post }_{8} \\
\triangle \%\end{array}$ & $\begin{array}{c}178 \pm 16 \\
178 \pm 24 \\
0\end{array}$ & $\begin{array}{c}226 \pm 21 \\
185 \pm 17^{* *} \\
-18(p=0,01)\end{array}$ & $\begin{array}{c}226 \pm 17 \\
215 \pm 25 \\
-5\end{array}$ & $\begin{array}{c}171 \pm 24 \\
170 \pm 33 \\
-1\end{array}$ & 0,0001 \\
\hline C-LDL (mg/dl) & $\begin{array}{l}\text { Pre }_{0} \\
\text { Post }_{8} \\
\triangle \%\end{array}$ & $\begin{array}{c}108 \pm 21 \\
107 \pm 24 \\
-0,9\end{array}$ & $\begin{array}{c}125 \pm 21 \\
110 \pm 17 \\
-12\end{array}$ & $\begin{array}{c}136 \pm 28 \\
131 \pm 24 \\
-1\end{array}$ & $\begin{array}{c}96 \pm 22 \\
95 \pm 29 \\
-1\end{array}$ & 0,0030 \\
\hline C-HDL (mg/dl) & $\begin{array}{l}\text { Pre }_{0} \\
\text { Post }_{8} \\
\triangle \%\end{array}$ & $\begin{array}{l}51 \pm 10 \\
59 \pm 14 \\
+16\end{array}$ & $\begin{array}{c}48 \pm 10 \\
58 \pm 9 * \\
+23(p=0,05)\end{array}$ & $\begin{array}{c}48 \pm 9 \\
57 \pm 14 \\
+17\end{array}$ & $\begin{array}{c}53 \pm 7 \\
60 \pm 7 \\
+15\end{array}$ & 0,6170 \\
\hline $\mathrm{TG}(\mathrm{mg} / \mathrm{dl})$ & $\begin{array}{l}\text { Pre }_{0} \\
\text { Post }_{8} \\
\triangle \%\end{array}$ & $\begin{array}{c}101 \pm 33 \\
91 \pm 26 \\
-5\end{array}$ & $\begin{array}{c}139 \pm 32 \\
99 \pm 23^{* *} \\
-27(p=0,01)\end{array}$ & $\begin{array}{c}174 \pm 81 \\
146 \pm 75 \\
-18\end{array}$ & $\begin{array}{c}105 \pm 41 \\
86 \pm 15 \\
-10\end{array}$ & 0,0122 \\
\hline
\end{tabular}

Los datos son presentados como media \pm desviación estándar. Grupos son descritos como; (HG): hiperglicémicos, (HC): hipercolesterolémicos, $(\mathrm{HG} / \mathrm{HC})$ : hiperglicémicos/hipercolesterolémicos y (SANOS): sujetos sanos o también control. Variables son descritas como; (CT): colesterol total, (C-LDL): lípidos de baja densidad, (C-HDL): lípidos de alta densidad, (TG): triglicéridos.

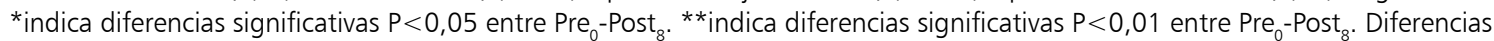
entre grupos fueron analizadas con ANOVA de 1 vía, mientras que las diferencias en el tiempo en cada grupo mediante ANOVA de medidas repetidas (grupos $x$ tiempo). 
lación entre variables. El nivel para significancia estadística se estableció en $p<0,05$. Todos los análisis estadísticos se realizaron utilizando el software STATISTICA (versión 8.0, StatSoft, Inc, Oklahoma, USA).

\section{Resultados}

Antes de la intervención existieron diferencias significativas $(\mathrm{p}<0,05)$ sólo en las variables glicemia, CT, LDL y TG, (Tabla 4).

Se presentó una reducción significativa $(p<0,01)$ en el peso corporal e IMC en los grupos $\mathrm{HC}, \mathrm{HG} / \mathrm{HC}$ y SANOS. El perímetro de cintura se modificó significativamente $(\mathrm{p}<0,01)$ en el grupo HG. No se observaron cambios significativos en los pliegues adiposos, excepto por una disminución del pliegue cresta iliaca en el grupo HC (Tabla 1).

La PAS se redujo significativamente $(\mathrm{p}<0,05)$ en el grupo $\mathrm{HC}$, así como la $\mathrm{PAD}$ en el grupo SANOS $-2,8 \%$. La frecuencia cardiaca de reposo se redujo $(\mathrm{p}<0,01)$ en el grupo $\mathrm{HC}$. El rendimiento físico aeróbico se incrementó significativamente $(p<0,01)$ en todos los grupos (Tabla 2).

La glicemia disminuyó significativamente $(\mathrm{p}<0,01)$ en los grupos HG e HG/HC, así como la insulina en los cuatro grupos de intervención (Tabla 4). Similarmente el HOMA IR $_{\text {presentó }}$ una reducción significativa en todos los grupos (Figura 1).

El CT y los triglicéridos disminuyeron significativamente $(p<0,01)$ en el grupo HC (Tabla 4$)$. El C-HDL se incrementó $(\mathrm{p}<0,05)$ en el grupo HC (Tabla 4).

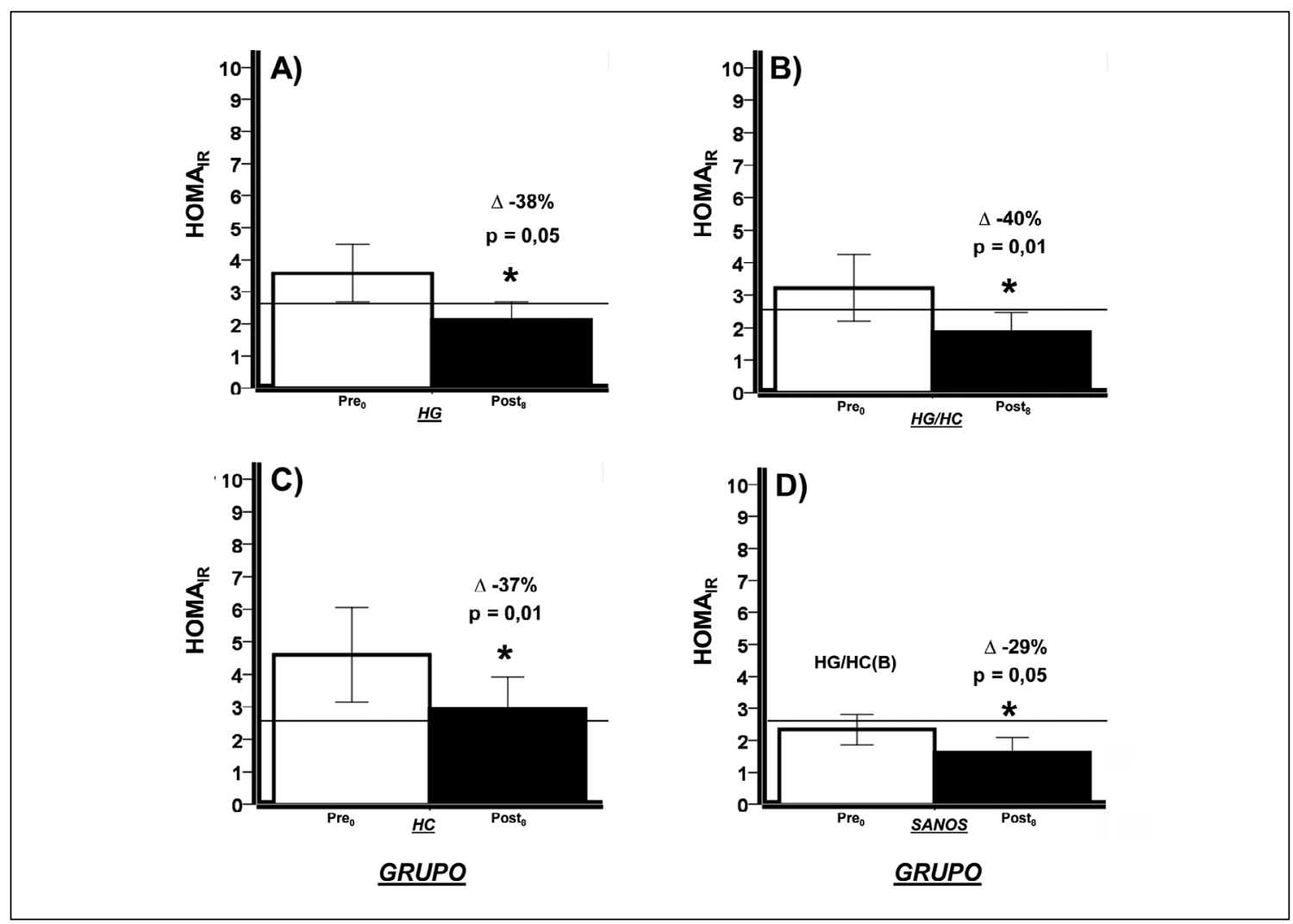

Figura 1. Cambios en $\mathrm{HOMA}_{\mathrm{IR}}$ en cuatro grupos de sujetos mujeres, sometidos a 8 semanas de ejercicio físico combinado. A: grupo hiperglicémicos (HG). B: grupo hiperglicémicos/hipercolesterolémicos (HG/HC). C: grupo hipercolesterolémicos (HC) y D: grupo de sujetos sanos (SANOS) o controles. Valores se presentan en media \pm error estándar entre antes $\left(P_{0} e_{0}\right)$ y después de intervención $\left(\right.$ Post $_{8}$ ). Línea continua entre barras indica valores límites standard de $\mathrm{HOMA}_{\mathrm{R}}$ de acuerdo a una población adulta

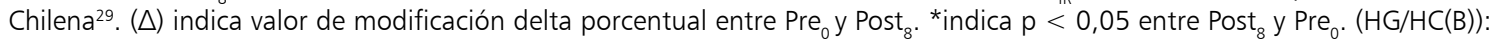
indica diferencias significativas $\mathrm{p}<0,05$, respecto a grupo $\mathrm{HG} / \mathrm{HC}$. 


\section{Discusión}

Los mayores hallazgos de este estudio indican que 8 semanas de EF combinado (i) disminuyen los niveles de insulino resistencia (Figura 1), y (ii) mejoran significativamente el rendimiento físico aeróbico de mujeres con y sin alteraciones metabólicas, (Tabla 2). Beneficios adicionales otorgan estos programas en la modificación favorable de la composición corporal (Tabla 1).

La reducción significativa de la glicemia en los grupos con valores previamente alterados $\mathrm{HG}$ -12 y HG/HC $-14 \%$ y en la insulina -30 y $-26 \%$ respectivamente para ambos grupos, indica un mayor control metabólico de la glucosa, siendo ello bien relacionado con mejoras en la insulino sensibilidad en estudios de $2^{9}$ y 12 semanas de $\mathrm{HIT}^{14}$, aunque nosotros observamos un plus en el presente protocolo de menor duración (8 semanas) respecto a otros de mayor duración, pues en un primer reporte Álvarez y cols ${ }^{14}$ aplicando HIT+EFS (60 min/sesión por 3 sesiones/semana= 3 meses) disminuyeron entre $\sim 4,2$ y $7 \%$ la glicemia de sujetos con hiperglicemia, así como entre 8 y $38 \%$ los valores de $\mathrm{HOMA}_{\mathrm{IR}}$. Los mismos autores en otro estudio de similar duración aplicando HIT $(20 \mathrm{~min} /$ sesión por 5 sesiones/semana $=3$ meses) ${ }^{18}$, reportaron una disminución de $\sim 16$ y $\sim 20 \%$ en la glicemia de sujetos hiperglicémicos y de hiperglicémicos/hipercolesterolémicos respectivamente, existiendo también una menor reducción en sujetos con glicemia normal. Tal parece que suele ser repetitivo que pacientes con línea de base alterada tiendan a responder mejor o en mayor grado al tratamiento con ejercicio vs. aquellos sanos, dilucidándose así cierta capacidad predictiva en la mejora del control metabólico en este tipo de pacientes.

Los grupos que presentaron una mayor reducción en el $\mathrm{HOMA}_{\mathrm{IR}}$ fueron aquellos tres con alteraciones metabólicas (HG, HC, y HG/HC) en rango -37 a $40 \%$ (Figura 1A, 1B y 1C). En estudios similares pero de menor duración, Whyte y cols $^{12}$ demostraron en 2 semanas de HIT, reducir los niveles de glicemia -3\% e insulina $-25 \%$. En el presente estudio, nosotros estimamos que los sujetos que no alcanzaron los valores de línea de normalidad estándar de acuerdo a un parámetro de la población Chilena $\left(\mathrm{HOMA}_{\mathrm{IR}}=2,6\right)^{28}$, pueden estar supeditados a requerir a) mayor tiempo $u$ volumen de ejercicio, b) otra metodología de ejer- cicio u c) ser considerados "bajos respondedores" al ejercicio de acuerdo al algunos polimosrfismos genéticos encontrados ${ }^{29}$.

Si bien es cierto el HOMA $\mathrm{IR}_{\mathrm{I}}$ ha presentado una correlación importante con la prueba gold estándar para determinar IR como el clamp euglicémico/hiperinsulinémico en sujetos diabéticos y no diabéticos ${ }^{30}$, considerando que en condiciones de ayuno es el hígado el principal órgano controlador de la glicemia y no tanto así el tejido muscular (que da cuenta del más de $75 \%$ de captación de glucosa vía insulino-estimulada ${ }^{7}$ ), los positivos resultados encontrados en la reducción del $\mathrm{HOMA}_{\mathrm{IR}}$ en este estudio, pueden reflejar más bien una reducción de la IR a nivel hepática, pudiendo ser explicados estos resultados por la reducción en algunos marcadores de grasa ectópica que de acuerdo a estudios previos, también se han relacionado con la reducción de pliegues de grasa subcutánea ${ }^{31}$.

Es importante mencionar que la distribución central del tejido adiposo ha sido relacionada también con un mayor índice de morbi-mortalidad ${ }^{32}$, por ello la disminución del perímetro de cintura resultante en el grupo HG -3,9\%, creemos que representa una disminución importante del riesgo cardiometabólico en estos pacientes. Estudios previos de HIT de $2^{12}, 8^{31}$ y $16^{33}$ semanas, han reportado disminuciones promedio del perímetro de cintura en rango de 1 a $7 \%(\sim 1$ a $5 \mathrm{~cm})$, asociándose estos cambios con el aumento de la sensibilidad de la insulina ${ }^{12,34}$. Adicionalmente, considerando que el pliegue adiposo que presentó mayor reducción fue la cresta iliaca (Figura 1), ello sugiere una plasticidad diferencial del tejido adiposo en respuesta al ejercicio físico, lo que coincide con resultados de recientes investigaciones ${ }^{35}$.

Previamente protocolos de HIT utilizados por otros grupos de investigación, han mostrado incrementos del C-HDL en respuesta a $\mathrm{HIT}^{36}$. En relación al C-LDL protocolos de HIT no han mostrado cambios $^{36,37}$. Kessler y cols $^{38}$ concluyeron que para producir efectos en el perfil lipídico, un programa de HIT debe tener una duración de al menos 8 semanas, siendo aún poco claras las adaptaciones a nivel de lipoproteínas plasmáticas. Interesantemente en este estudio, la reducción del peso corporal no se correlacionó significativamente con ninguna de las modificaciones plasmáticas.

$\mathrm{Al}$ respecto la metodología de ejercicio tipo HIT, cabe destacar que el ejercicio físico de corta duración, bajo volumen ( $\leq 12$ semanas) y alta in- 
Ejercicio físico combinado mejora el control metabólico - C. Álvarez et al

tensidad de ejercicio (80-100 frecuencia cardiaca reserva), pero de carácter intermitente (30 seg-1 min trabajo/1 a 2 min de recuperación), pueden ser tan efectivos en la mejora del control metabólico de la glucosa y sensibilidad insulínica en relación al ejercicio continuo de baja intensidad y larga duración según se ha reportado ${ }^{39,40}$, haciéndose muy importante, primeramente la aplicación de un riguroso screening clínico antes de la inclusión de pacientes en estos programas así como también una fase de adaptación cardiovascular al ejercicio que en cierta medida de acuerdo a nuestra experiencia da garantía a una buena tolerancia a la intervención.

Por otra parte, nosotros encontramos una disminución importante en la presión arterial sistólica de sujetos con hipercolesterolemia PAS 122 antes y después $111 \mathrm{~mm} / \mathrm{Hg}$, y un incremento en la performance aeróbica valorada a través de la prueba de caminata TUKK, validado en la población Chilena ${ }^{24}$. Sin duda, ambas modificaciones vienen similarmente en aportar en la disminución en los factores de riesgo cardiovascular característica en la población Chilena que sufre sobrepeso/ obesidad.

Este es el primer estudio de intervención en pacientes de la salud pública en Chile, de menor tiempo de duración aplicando un programa de ejercicio físico combinado (HIT+EFS) sobre el comportamiento de variables metabólicas, cardiovasculares, antropométricas y de condición física en sujetos con alteraciones metabólicas frecuentes, donde nosotros mostramos efectos benéficos de estos programas cortos a modo que puedan ser aplicados en centros de atención primaria de salud como herramienta "preventiva y terapéutica" en pacientes sanos y patológicos, donde concluimos que las adaptaciones más benéficas del estos programas atañen a pacientes con IR y dislipidemias.

Agradecimientos: A todos los co-autores que hicieron posible que el estudio se llevara a cabo. A Carmen Flores, Directora del CESFAM Los Lagos, por su inestimable apoyo en la instauración del programa de ejercicio físico.

\section{Referencias}

1. Abdul-Ghani MA, DeFronzo RA. Pathogenesis of Insulin Resistance in Skeletal Muscle. J Biomed Biotechnol 2010; 2010.
2. Petersen KF, Shulman GI. Etiology of Insulin Resistance. The American journal of medicine 2006; 119(5): S10-S6.

3. Minsal. Ministerio de Salud. Gobierno de Chile. Encuesta Nacional de Salud ENS Chile 2009-2010. Disponible en: http://www.redsalud.gov.cl/portal/url/item/99c12b8 9738d80d5e04001011e0113f8.pdf 2011.

4. Colberg SR, Sigal RJ, Fernhall B, Regensteiner JG, Blissmer BJ, Rubin RR, et al. Exercise and Type 2 Diabetes: The American College of Sports Medicine and the American Diabetes Association: joint position statement. Diabetes Care 2010; 33(12): e147-e67.

5. Ferguson MA, Alderson NL, Trost SG, Essig DA, Burke JR, Durstine JL. Effects of four different single exercise sessions on lipids, lipoproteins, and lipoprotein lipase. J Appl Physiol 1998; 85(3): 1169-74.

6. Terada T, Friesen A, Chahal BS, Bell GJ, McCargar LJ, Boul, et al. Exploring the Variability in Acute Glycemic Responses to Exercise in Type 2 Diabetes. Journal of Diabetes Research 2013; 2013: 6.

7. DeFronzo RA. Glucose Intolerance and Aging: Evidence for Tissue Insensitivity to Insulin. Diabetes 1979; 28 (12): 1095-101.

8. Booth FW, Chakravarthy MV, Gordon SE, Spangenburg EE. Waging war on physical inactivity: using modern molecular ammunition against an ancient enemy. J Appl Physiol 2002; 93(1): 3-30.

9. Babraj J, Vollaard N, Keast C, Guppy F, Cottrell G, Timmons J. Extremely short duration high intensity interval training substantially improves insulin action in young healthy males. BMC Endocrine Disorders 2009; 9 (1): 3.

10. Little JP, Gillen JB, Percival ME, Safdar A, Tarnopolsky MA, Punthakee Z, et al. Low-volume high-intensity interval training reduces hyperglycemia and increases muscle mitochondrial capacity in patients with type 2 diabetes. J Appl Physiol 2011; 111 (6): 1554-60.

11. Talanian JL, Holloway GP, Snook LA, Heigenhauser GJF, Bonen A, Spriet LL. Exercise training increases sarcolemmal and mitochondrial fatty acid transport proteins in human skeletal muscle. Am J Physiol-Endocrinol Metab 2010; 299 (2): E180-E8.

12. Whyte LJ, Gill JMR, Cathcart AJ. Effect of 2 weeks of sprint interval training on health-related outcomes in sedentary overweight/obese men. Metabolism 2010; 59 (10): 1421-8.

13. Boutcher SH. High-Intensity Intermittent Exercise and Fat Loss. Journal of Obesity 2011; 2011.

14. Álvarez C, Ramírez R, Flores M. Zúñiga C, Celis-Morales CA. Effect of sprint interval training and resistance exercise on metabolic markers in overweight women. Rev Med Chile 2012; 140 (10): 1289-96.

15. Izquierdo M, Ibáñez J, González-Badillo JJ, Häkkinen K, Ratamess NA, Kraemer WJ, et al. Differential effects of strength training leading to failure versus not to failure on hormonal responses, strength, and muscle power gains. J Appl Physiol 2006; 100 (5): 1647-56.

16. Holten MK, Zacho M, Gaster M, Juel C, Wojtaszewski JFP, Dela F. Strength Training Increases Insulin-Me- 
diated Glucose Uptake, GLUT4 Content, and Insulin Signaling in Skeletal Muscle in Patients With Type 2 Diabetes. Diabetes 2004; 53 (2): 294-305.

17. Álvarez C, Campillo RR. Effects of a low intensity strength training program on overweight/obese and premenopausal/menopausal women. Revista Brasileira de Cineantropometria \& Desempenho Humano 2013; 15: 427-36.

18. Álvarez C, Ramírez-Campillo R, Flores-Opazo M, Henríquez-Olguín $\mathrm{C}$, Campos $\mathrm{C}$, Carrasco $\mathrm{V}$, et al. Metabolic response to high intensity exercise training in sedentary hyperglycemic and hypercholesterolemic women. Rev Med Chile 2013; 141: 1293-99.

19. Celis-Morales CA, Pérez-Bravo F, Ibáñez L, Salas C, Bailey ME, Gill JM. Objective vs self-reported physical activity and sedentary time: effects of measurement method on relationships with risk biomarkers. PloS one. 2012; 7(5): e36345.

20. WHO. Definition, Diagnosis and Classification of Diabetes Mellitus and its Complications; Report of a WHO Consultation Part 1: Diagnosis and Classification of Diabetes Mellitus. WHO. 1999.

21. NCEP. Executive summary of the third report of the national cholesterol education program (ncep) expert panel on detection, evaluation, and treatment of high blood cholesterol in adults (adult treatment panel iii). JAMA-J Am Med Assoc 2001; 285(19): 2486-97.

22. Marfell-Jones M, Olds T, Stewart A, Carter L. International standards for anthropometric assessment: ISAK 1st ed. Potchefstroom, South Africa: The International Society for the Advancement of Kinanthropometry (ISAK); 2006.

23. Laukkanen RM, Kukkonen-Harjula TK, Oja P, Pasanen ME, Vuori IM. Prediction of change in maximal aerobic power by the $2-\mathrm{km}$ walk test after walking training in middle-aged adults. Int J Sports Med 2000; 21 (2): 113-6.

24. Díaz E, Saavedra C. Evaluación de la condición física en adultos Chilenos. Santiago: IND, Instituto Nacional de deportes; 2008.

25. Karvonen J, Vuorimaa, T. Heart rate and exercise intensity during sports activities. Practical application. Sports Med 1988; 5 (5): 303-11.

26. Matthews DR, Hosker JP, Rudenski AS, Naylor BA, Treacher DF, Turner RC. Homeostasis model assessment: insulin resistance and $\beta$-cell function from fasting plasma glucose and insulin concentrations in man. Diabetologia 1985; 28 (7): 412-9.

27. Friedewa.Wt, Fredrick.Ds, Levy RI. Estimation of concentration of low-density lipoprotein cholesterol in plasma, without use of preparative ultracentrifuge. Clin Chem 1972; 18 (6): 499-\&.

28. Acosta BAM, Escalona OM, Maiz GA, Pollak CF, Leighton F. Determinación del índice de resistencia insulínica mediante HOMA en una población de la Región Metropolitana de Chile. Rev Med Chile 2002; 130: 1227-31.

29. Davidsen PK, Gallagher IJ, Hartman JW, Tarnopolsky
MA, Dela F, Helge JW, et al. High responders to resistance exercise training demonstrate differential regulation of skeletal muscle microRNA expression. J Appl Physiol 2011; 110 (2): 309-17.

30. Ikeda Y, Suehiro T, Nakamura T, Kumon Y, Hashimoto $\mathrm{K}$. Clinical significance of the insulin resistance index as assessed by homeostasis model assessment. Endocr J 2001; 48 (1): 81-6.

31. Mourier A, Gautier J-F, Kerviler ED, Bigard AX, Villette J-M, Garnier J-P, et al. Mobilization of Visceral Adipose Tissue Related to the Improvement in Insulin Sensitivity in Response to Physical Training in NIDDM: Effects of branched-chain amino acid supplements. Diabetes Care. 1997; 20 (3): 385-91.

32. Wright ME, Chang SC, Schatzkin A, Albanes D, Kipnis V, Mouw T, et al. Prospective study of adiposity and weight change in relation to prostate cancer incidence and mortality. Cancer 2007; 109 (4): 675-84.

33. Tjønna AE, Lee SJ, Rognmo $\varnothing$, Stølen TO, Bye A, Haram PM, et al. Aerobic Interval Training Versus Continuous Moderate Exercise as a Treatment for the Metabolic Syndrome. Circulation 2008; 118 (4): 346-54.

34. Mourier A, Gautier JF, De Kerviler E, Bigard AX, Villette JM, Garnier JP, et al. Mobilization of visceral adipose tissue related to the improvement in insulin sensitivity in response to physical training in NIDDM. Effects of branched-chain amino acid supplements. Diabetes Care 1997; 20 (3): 385-91.

35. Ramírez-Campillo R, Andrade DC, Campos-Jara C, Henríquez-Olguín C, Alvarez-Lepín C, Izquierdo M. Regional fat changes induced by localized muscle endurance resistance training. J Strength Cond Res / National Strength \& Conditioning Association 2013; 27 (8): 221924.

36. Schjerve IE, Tyldum GA, Tjonna AE, Stolen T, Loennechen JP, Hansen HE, et al. Both aerobic endurance and strength training programmes improve cardiovascular health in obese adults. Clin Sci (Lond) 2008; 115(9): 283-93.

37. Heydari M, Freund J, Boutcher SH. The Effect of HighIntensity Intermittent Exercise on Body Composition of Overweight Young Males. Journal of Obesity 2012; 2012: 8 .

38. Kessler H, Sisson, SB., Short, KR. The Potential for High-Intensity Interval Training to Reduce Cardiometabolic Disease Risk. Sports Medicine 2012; 42(6): 489-509.

39. Heydari M, Freund J, Boutcher SH. The effect of highintensity intermittent exercise on body composition of overweight young males. Journal of obesity 2012; 2012: $1-8$.

40. Tjonna AE, Lee SJ, Rognmo O, Stolen TO, Bye A, Haram PM, et al. Aerobic interval training versus continuous moderate exercise as a treatment for the metabolic syndrome: a pilot study. Circulation 2008; 118 (4): 346-54. 